\title{
NON-PERTURBATIVE INTERACTIONS IN STRING THEORY
}

UPR-524T

\author{
Ram Brustein and Burt A. Ovrut \\ Department of Physics \\ University of Pennsylvania \\ Philadelphia, PA 19104
}

\begin{abstract}
New non-perturbative interactions in the effective action of two dimensional string theory are described. These interactions are due to "stringy" instantons.
\end{abstract}

\section{INTRODUCTION}

Non perturbative effects in string theory are important. To someone familiar with weakly coupled quantum field theories that may sound a little strange. After all, nonperturbative effects are much smaller than perturbative effects in such theories. Typically, in a weakly coupled field theory, nonperturbative effects have strength $e^{-1 / g^{2}}$ compared to perturbative quantities that have strength $g^{2}$, where $g$ is the coupling parameter of the theory. For example if $g^{2}=.3$ the ratio of these numbers is about $1: 100$. The only case in which non-perturbative quantities are important is when their value in perturbation theory vanishes. This is the situation in superstring theory for a number of important quantities, e.g., the size of supersymmetry breaking ${ }^{(1)}$. The smallness of nonperturbative effects is actually an advantage in string theory because it may explain the small ratio of the electroweak scale and Planck scale.

${ }^{*}$ Supported in part by the Department of Energy under contract No. DOE-AC02-76-ERO-3071.

Talk presented by B. Ovrut at the XXVI International Conference on High Energy Physics, Aug. 1992, Dallas, Texas .
To confront string theory with the real observable world we have to understand the source of non-perturbative interactions in string theory and know how to calculate them. Matrix models, and especially $d=1$ matrix models ${ }^{(2)}$, offer a unique opportunity to obtain some insight into non-perturbative string theory. Certain matrix models have associated with them very simple string theories with a low number of degrees of freedom, propagating in a low number of space-time dimensions. The $d=1$ matrix model is the most complicated matrix model which can still be exactly solved. On the other hand, it describes the simplest space-time dynamics which is still interesting. In the double scaling limit, the $d=1$ matrix model describes strings propagating in one time dimension and one spatial dimension. An equivalent description is given in terms of a bosonic collective field theory ${ }^{(3)}$ in $1+1$ dimensions of one massless field. Notable features of collective field theory is that the kinetic energy is not canonical and the theory is not Lorentz invariant.

The $d=1$ matrix models, or the equivalent field theories have the power to describe non-perturbative phenomena in the associated $1+1$ string theories. This is interesting by it- 
self. However, there may well be some general features of non-perturbative string theory that are common to all string theories, including more complicated theories in higher dimensions such as $d=4$. By studying the generic features of non-perturbative behaviour in $1+1$ dimensional string theories, as we do in this talk, one may learn about more realistic 4-dimensional string theories. It is of interest to ask whether or not there is any indication in string theory of common, non-perturbative behaviour. The answer ${ }^{(4)}$ is yes!

First recall that in quantum field theory there is a well known connection between the large order behaviour of amplitudes and nonperturbative effects. Typically, amplitudes grow as $G$ ! where $G$ is the number of loops, while non-perturbative effects have strength $e^{-1 / g^{2}}$, where $g$ is the coupling constant of the theory. Both of these facts follow from the existence of non-trivial classical solutions of the equations of motion of the field theory in Euclidean space, i.e. instantons. The magnitude of the non-perturbative effects due to non-trivial solutions in a field theory with one dimensionless coupling parameter $g$ can be estimated using a simple scaling argument. Since the coupling parameter in this case can be scaled away, the action can be written as $S(\phi, g)=1 / g^{2} \widetilde{S}(\widetilde{\phi})$, where $\widetilde{S}$ does not depend on $g$. Therefore, any classical Euclidean solution with finite action has an action of order $1 / g^{2}$. The magnitude of large order terms in the perturbative expansion can also be estimated by counting Feynman diagrams. The number $G$ ! basically comes from the number of diagrams.

Large order growth of perturbative amplitudes is a common feature of matrix models and more complicated string theories ${ }^{(4)}$. For a review of large order behaviour of matrix model amplitudes see ref.(5). All matrix models, as well as critical bosonic string theory in 26 dimensions, exhibit a strange phenomenon. The magnitude of $G^{\prime}$ th order amplitudes in perturbation theory grow like $(2 G)$ !. It turns out that, in much the same way as $G$ ! behaviour corresponds to $e^{-1 / g^{2}}$ nonperturbative effects in quantum field theory, in matrix models the large order $(2 G)$ ! behaviour, would correspond to non-perturbative effects of strength $e^{-1 / g}$. How do these peculiar effects arise? In matrix models, there is a new type of instanton, involving a single eigenvalue, that is responsible for these effects.

We expect that string theory is described at low energies by an effective field theory with one dimensionless coupling parameter. In view of the above scaling argument in quantum field theory, it is of interest to ask how an instanton action of order $1 / g$ can ever arise, in such an effective field theory. We found that in matrix models, the associated effective action does not obey the same scaling argument, $S(\phi, g) \neq 1 / g^{2} \widetilde{S}(\widetilde{\phi})$. Instead, one finds that $g$ cannot be completely scaled out of $\widetilde{S}$ due to "scale breaking terms". That is $S(\phi, g)=1 / g^{2} \widetilde{S}(\widetilde{\phi}, g)$. It follows that a nontrivial solution can be a function of $g$. Furthermore, if for such a solution $\widetilde{S} \sim g$, then $S \sim 1 / g$. This is exactly what happens for one eigenvalue instantons.

In this talk, the role of these "stringy" instantons in the effective field theory of string theory is discussed and the non-perturbative interactions that they produce are derived. For a more detailed and extensive discussion of the issues in this talk, as well as a comprehensive list of references, the interested reader is refered to the original papers ${ }^{(6)}$.

\section{EFFECTIVE FIELD THEORY FOR STRINGS}

Collective field theory for $d=1$ matrix models is written in terms of the density of eigenvalues, $\phi(x, t)=\sum \delta\left(x-\lambda_{i}(t)\right)$ where $\lambda_{i}$ are the eigenvalues of the matrix. The size of the matrix, $N$, is very large. The field $\phi$ is called the collective field. The Lagrangian density of this collective field theory is

$$
\mathcal{L}_{\text {eff }}=\frac{1}{2} \frac{\int^{x} \dot{\phi} \int^{x} \dot{\phi}}{\phi}-\frac{\pi^{2}}{6} \phi^{3}-\frac{1}{2}\left(\frac{1}{g}-x^{2}\right) \phi
$$

It is obvious that the field $\phi$ does not have canonical kinetic energy and that the theory is 
not Lorentz invariant. These deficiencies will be corrected soon. For a review of perturbative results of collective field theory see ref.(7). The classical equations of motion are not the usual field equations ${ }^{(6)}$. Instead they are

$\partial_{x}\left(\int d y \partial_{t} \frac{\int^{y} \dot{\phi}}{\phi}-\frac{\int^{x} \dot{\phi} \int \dot{\phi}}{2 \phi^{2}}-\frac{\pi^{2}}{2} \phi^{2}-\frac{1}{2}\left(\frac{1}{g}-x^{2}\right)\right)_{\mid x=\lambda_{i}(t)}=0$

where the index $i$ now runs over $i=1, \ldots, N \rightarrow$ $\infty$.

These equations allow solutions of high density regions where $\phi>>1$ and low density regions. The static, high density, solution of these equations is very simple

$$
\phi_{0}=\frac{1}{\pi} \sqrt{x^{2}-\frac{1}{g}}
$$

where $|x| \geq \sqrt{\frac{1}{g}}$. This static solution is displayed in Figure 1.

Figure 1. The potential and static, high density, solution of collective field theory.

There are also interesting time dependent Euclidean solutions to Eq.(2).

$$
\phi_{\text {inst }}(x, \theta)=\delta\left(x-\frac{1}{\sqrt{g}} \cos \left(\theta-\theta_{0}\right)\right)
$$

This is an instanton that corresponds to tunneling of one eigenvalue across the barrier from $x=\sqrt{\frac{1}{g}}$ at Euclidean time $\theta=\theta_{0}$ to $x=-\sqrt{\frac{1}{g}}$ at $\theta=\theta_{0}+\pi$. Note that the classical solution in Eq.(4) is not a solution of the Euclidean continuation of of the unconstrained field theory equations of motion. The action of this one eigenvalue instanton is $\pi / g$, as mentioned in the introduction.

The collective field theory Lagrangian density, expression (2), has two notable deficiencies. First, the kinetic energy term is not in canonical form. This means that we have not identified correctly the canonical field of the theory. Second, and more important, the coordinate $x$ appears in the potential energy and therefore Lorentz invariance seems to be broken explicitly. We remove both deficiencies. The first, following ref.(3), by a field redefinition $\partial_{x} \zeta=\phi-\phi_{0}$ and a coordinate redefinition $x \rightarrow \tau=\frac{1}{\pi} \int^{x} \frac{d y}{\phi_{0}}$. The second, following ref.(8), by enlarging the theory to include a new field, $D$. The non-trivial vacuum expectation value of this new field is responsible for the spontaneous breaking of Lorentz invariance. The resulting action for the fields $D, \zeta$ in the new coordinates is

$$
\begin{aligned}
& \mathcal{S}=\int d t d \tau\left\{\frac{1}{2} \frac{\nabla \zeta \cdot \nabla \zeta}{1+2 \sqrt{\pi} \frac{e^{D}}{\left(1-\frac{1}{g} e^{D}\right)^{2}} \nabla \zeta \cdot \nabla D}\right. \\
& -\frac{\sqrt{\pi}}{4} \frac{e^{D}}{\left(1-\frac{1}{g} e^{D}\right)^{2}} \frac{(\nabla \zeta \cdot \nabla D)^{3}}{1+2 \sqrt{\pi} \frac{e^{D}}{\left(1-\frac{1}{g} e^{D}\right)^{2}} \nabla \zeta \cdot \nabla D} \\
& -\frac{\sqrt{\pi}}{12} \frac{e^{D}}{\left(1-\frac{1}{g} e^{D}\right)^{2}}(\nabla \zeta \cdot \nabla D)^{3} \\
& \left.-\frac{1}{384 \pi} e^{-2 D}\left[1-\frac{1}{g} e^{D}\right]^{4}\left[(\nabla D)^{2}-4\right]\right\}
\end{aligned}
$$

The kinetic energy for $\zeta$ is clearly canonical, and Lorentz invariance is manifest. The action is also invariant under an unexpected $^{(9)}$ shift symmetry $\zeta \rightarrow \zeta+$ const. The field $\zeta$ has no potential, only derivative interactions. This is similar to the dilaton interactions in the critical bosonic string and the corresponding complex field, $S$, in the superstring. The parameter $g$ appears in the action (5) only through the coupling parameter of the effective theory, which is $g(D)=4 \sqrt{\pi} \frac{e^{D}}{\left(1-\frac{1}{g} e^{D}\right)^{2}}$. Thus $g$ cannot be absorbed into a redefinition of $D$ and scaled 
away from the effective action. The coupling parameter $g(D)$ is an example of the "scale breaking" terms that were discussed in the introduction.

The general solution of the equations of motion derived from (5) is given by

$$
<D>=a(t-\bar{t})+b(\tau-\bar{\tau}), \quad<\zeta>=\frac{1}{g}+c
$$

where $a, b, c, \bar{t}$ and $\bar{\tau}$ are real parameters, $b^{2}-$ $a^{2}=4$ and $c, \bar{t}, \bar{\tau}$ are arbitrary. An interesting vacuum solution, which is a combination of two solutions is $\langle\zeta\rangle=\frac{1}{g}$ and $\langle D\rangle=-2 \tau$ for $\tau \geq \ln \sqrt{\frac{1}{g}}$, henceforth called region $I$, and $<D>=-2\left(\tau-2 \ln \sqrt{\frac{1}{g}}+\pi\right)$ for $\tau \leq \ln \sqrt{\frac{1}{g}}-$ $\pi$, henceforth called region $I I I$. The effective coupling parameter in region $I$ is $g_{-}$and in region $I I I$ is $g_{+}$. The spatial interval $\ln \sqrt{\frac{1}{g}}-$ $\pi \leq \tau \leq \ln \sqrt{\frac{1}{g}}$ is called region $I I$. We plot the effective coupling parameters in Figure 2.

Figure 2. Effective coupling parameters in different regions of space.

The two dashed "walls" in Figure 2 mark the boundaries of the regions. As can be seen from Figure 2, in the regions far away from the two walls, the coupling parameters are small and we expect the effective field theory (5) to provide a good description of physics. Near the "walls" the coupling parameters blow up. Region $I I$ is unknown territory.

We use low density collective field theory as a guide in the unknown territory, region $I I$.
Comparing the low density collective field theory solution to the solution of the effective field theory we see that in region $I I$ their solutions should be the same.

Figure 3. Instanton tunneling between regions $I$ and III. The static solutions are also shown.

In Euclidean space the solution is therefore the instanton of Eq.(4), expressed in the new coordinates

$\phi_{\text {inst }}(\tau, \theta)=\frac{1}{\sin \left(\theta-\theta_{0}\right)} \delta\left(\tau-\left[\ln \sqrt{\frac{1}{g}}-\left(\theta-\theta_{0}\right)\right]\right)$

This is an instanton which corresponds to the tunneling of a single eigenvalue across the barrier from $\tau=\ln \sqrt{\frac{1}{g}}$ at $\theta=\theta_{0}$ to $\tau=\ln \sqrt{\frac{1}{g}}-\pi$ at $\theta=\theta_{0}+\pi$. Note that the velocity of the eigenvalue at either side of the barrier vanishes. Therefore, the Euclidean conjugate momentum of the instanton in region $I I$, matches continuously at the boundaries with the vanishing conjugate momentum of the static vacua $\phi_{0}$ in regions $I$ and $I I I$. We represent this tunneling process in Figure 3 .

The action (5) is the effective space-time action that corresponds to string theories de- 
scribed by the following $\sigma$-model ${ }^{(7,8)}$

$$
\begin{aligned}
& I=\frac{1}{4 \pi} \int d^{2} z \sqrt{\hat{g}}\left\{\hat{g}^{\alpha \beta} G_{\mu \nu} \partial_{\alpha} X^{\mu} \partial_{\beta} X^{\nu}+\right. \\
& \hat{R} D(X)+2 T(X)\}
\end{aligned}
$$

where $\hat{g}_{\alpha \beta}$ is the fixed world sheet metric with Euclidean signature and $\hat{R}$ is the corresponding Ricci scalar. The sigma model field $X_{\mu}$ stands for two scalar fields, $X_{0}(z)$, and $X_{1}(z)$. The field $G_{\mu \nu}(X)$ is the target space metric, assumed here to have Euclidean signature, $D(X)$ is the dilaton, and $T(X)$ is the tachyon. The field $\zeta$ is related to the tachyon $\zeta \propto T e^{-D}$. The instanton shown in Figure 3 is therefore a "stringy instanton".

\section{NON-PERTURBATIVE INTERACTIONS}

We integrate over the instantons and represent their effects as effective terms in the $D, \zeta$ theory. Since $\zeta$ is the light field we restrict our attention to $\zeta$ operators. The effective operators are especially important. They provide the bridge between the discrete sector of the theory and the continuous sector. A more detailed analysis is given in ref.(6).

The instanton has three parameters, $\bar{\tau}, \theta_{0}$ and $\alpha$. The parameters $\bar{\tau}$ and $\theta_{0}$ were defined in Eqs.(6) and (7). The parameter $\alpha$ is related to the parameter $a$ in the Euclidean space continuation of Eq.(6), $a=2 \sin \alpha$. Changing $\alpha$ results in the rotation of the vacuum solution in $\tau-\theta$ space. There are three zero modes corresponding to the three broken generators of the Euclidean group associated with $\bar{\tau}, \theta_{0}$, $\alpha$. These have to be integrated and produce a volume factor $V o l \propto \int d \bar{\tau} d \theta_{0} d \alpha$.

The dilute gas summation over instantons induces effective terms in the $D, \zeta$ Lagrangian. The most general action induced by instantons is $\Delta S=\int d \tau d \theta\left\{\sum_{n} C_{n} O_{n}(\tau, \theta)\right\}$, where $O_{n}$ are local operators built from $D$ and $\zeta$ and their derivatives. The coefficients $C_{n}$ can be computed by expanding the action around the instanton background. All the coefficients $C_{n}$ are proportional to the universal factor of the exponent of the instanton action and the remaining factor depends on the particular operator that is considered. Since the "size" of the instanton is $\sqrt{g}$, the dimension of the operator determines the $g$ dependence of $C_{n}$. That is

$$
C_{n}=\tilde{C}_{n} g^{d(n)} e^{-\frac{\pi}{g}}
$$

where $d(n)=\left[\operatorname{dimension}\left(O_{n}\right)\right]^{\frac{1}{2}}-1$ and $\tilde{C}_{n}$ is a numerical coefficient. The coefficient $\tilde{C}_{n}$ is not expected to be particularly large or particularly small.

We are interested in large $\frac{1}{g}$ that corresponds to small $g$. In that case the dominant and most interesting operator is the unit operator. All other operators are suppressed by powers of $g$. The coefficient of the unit operator is given by $C_{0}=\tilde{C}_{0} \frac{1}{g} e^{-\frac{\pi}{g}}$. This result was obtained in the background of a constant field $\langle\zeta\rangle=\frac{1}{g}$. Lorentz invariance then dictates that at least for slowly varying fields the effective operator depends on the full field $\zeta$ and not just its constant mode $\frac{1}{g}$. Therefore the final result of the induced operator is

$$
\Delta \mathcal{L}_{0}=\tilde{C}_{0} \zeta e^{-\pi \zeta}
$$

written in terms of the tachyon $T$ the induced operator is

$$
\Delta \mathcal{L}_{0}=\tilde{C}_{0} T e^{-D} e^{-\pi T e^{-D}}
$$

This operator breaks the $\zeta$ shift symmetry. It induces a runaway non-perturbative potential for the field $\zeta$. Similar effects due to field theoretic non-perturbative interactions occur in more complicated string theories ${ }^{(1)}$. Recall that in more complicated theories the dilaton has only derivative interactions and no potential. Known field theoretic non-perturbative interactions induce a runaway potential for the dilaton and break the dilaton shift symmetry. These effects, however, are of typical field theory strength i.e. $e^{1 / g^{2}}$. In the superstring, the appearance of a potential for the $S$ field signals supersymmetry breaking.

It is therefore tempting to conjecture that stringy instantons similar to our stringy 
instantons appear in 4-dimensional superstring theories and that they induce nonperturbative operators of the type discussed above. In that case these operators are expected to be proportional to the universal factor $e^{-\sqrt{S}}$, where $S$ is a complex field that naturally appears in the effective low energy supergravity field theory obtained from superstring theory. The dilaton is related to the real part of $S,<\operatorname{Re} S>\sim \frac{1}{g^{2}}$. Note that the non-perturbative effects considered previously in the literature induced operators of field theory strength, and therefore are proportional to the universal factor $e^{-S}$. Since the coupling parameter $g$ is expected to be small, the difference between these two universal factors can be big. If indeed such non-perturbative 'stringy" interactions occur, they may have important phenomenological consequences.

\section{References}

[1] L. Dixon, "Supersymmetry breaking in string theory", presented at the 15th APS Div. of Particles and Fields Conf., pp. 811-822, January 3-6, 1990.

[2] D. J. Gross, N. Miljkovic, "A nonperturbative solution of $\mathrm{d}=1$ string theory", Phys.Lett.B238, pp. 217-224, (1990);

P. Ginsparg and J. Zinn-Justin, "2-d gravity + 1-d matter", Phys. Lett.B240, pp. 333-340, (1990);

E. Brezin, V. Kazakov and Al. B. Zamolodchikov, "Scaling violation in a field theory of closed strings in one physical dimension", Nucl. Phys.B338, pp. 673-688, (1990).

[3] S. R. Das, and A. Jevicki, "String field theory and physical interpretation of $\mathrm{d}=$ 1 strings", Mod.Phys.Lett.A5, pp. 16391650, (1990).

[4] S. H. Shenker, "The strength of nonperturbative effects in string theory", presented at the Cargese Workshop on Random Surfaces, Quantum Gravity and
Strings, Cargese, France, May 28 - Jun 1, 1990.

[5] P. Ginsparg and J. Zinn- Justin, "Large order behavior of non-perturbative gravity" Phys. Lett. B255, pp. 189-196, (1991).

[6] R. Brustein and B. Ovrut, University of Pennsylvania preprints, UPR-522T and UPR-523T (1992).

[7] K. Demeterfi, A. Jevicki and J. P. Rodrigues, "Perturbative results of collective string field theory", Mod.Phys.Lett.A6, pp. 3199-3212, (1991).

[8] R. Brustein and S. P. De Alwis, "Background independent effective action from $\mathrm{d}=1$ matrix model", Phys.Lett.B272, pp. 285-289, (1991).

[9] T. Banks, "The tachyon potential in string theory", Nucl. Phys.B361, pp. 166172, (1991). 